\title{
Standing out in a networked communication context: Toward a network contingency model of public attention
}

new media \& society

(C) The Author(s) 2020 Article reuse guidelines: sagepub.com/journals-permissions DOI: I0.I I77/|46|444820939445 journals.sagepub.com/home/nms

@SAGE

\author{
Aimei Yang \\ University of Southern California, USA
}

\section{Adam J Saffer}

University of North Carolina, USA

\begin{abstract}
Social media can offer strategic communicators cost-effective opportunities to reach millions of individuals. However, in practice it can be difficult to be heard in these crowded digital spaces. This study takes a strategic network perspective and draws from recent research in network science to propose the network contingency model of public attention. This model argues that in the networked social-mediated environment, an organization's ability to attract public attention on social media is contingent on its ability to fit its network position with the network structure of the communication context. To test the model, we combine data mining, social network analysis, and machine-learning techniques to analyze a large-scale Twitter discussion network. The results of our analysis of Twitter discussion around the refugee crisis in 2016 suggest that in high core-periphery network contexts, "star" positions were most influential whereas in low core-periphery network contexts, a "community" strategy is crucial to attracting public attention.
\end{abstract}

\section{Keywords}

Big data, core-periphery structure, public attention, social network analysis

\section{Corresponding author:}

Aimei Yang, Annenberg School for Communication and Journalism, University of Southern California, 3630 Watt Way 324B, Los Angeles, CA 90089, USA.

Email: aimeiyan@usc.edu 


\section{Introduction}

Social media offer strategic communicators cost-effective opportunities to potentially reach millions of individuals (Guo and Saxton, 2018; Ihm, 2019). Yet, strategic communicators find in practice that being heard or "breaking through" the crowded mediated public sphere is difficult (Griffith, 2015). And when communicators do breakthrough, their messages may become distorted in communication environments where attention and meaning "combine to amplify, bury, or distort key messages" (Bennett et al., 2018: 662). Strategic communicators are not alone in these crowded mediated spaces; they are connected to others who may also play an influential role in how an organization attempts to attract public attention online.

Organizations, especially nongovernmental and nonprofit organizations (NGOs), often seek to gain influence and attract the limited supply of public attention (Sommerfeldt and Yang, 2017). Strategic communicators at NGOs know that public attention is critical for garnering public support and mobilizing resources. As Bernard Kouchner, founder of Médecins sans Frontières (MSF) famously put it during the Biafra crisis: "Where there is no camera, there is no humanitarian intervention" (Cate, 2002: 5). Being in the spotlight helps NGOs to raise funds, recruit volunteers/members, and gain access to different types of capital. NGOs that have stayed in the spotlight for extensive periods tend to emerge as issue leaders (e.g. Green Peace, Amnesty International, Doctors Without Bounders, etc.), and their agendas have better chances to influence public discourse (Shumate et al., 2005). Today, the spotlight that NGOs need to be in may not be coming from a camera but rather from the attention they are able to attract online.

This study takes a strategic network perspective (O'Connor and Shumate, 2018; Yang and Taylor, 2015) to understand how NGOs can attract public attention on social media by recognizing that communicators are situated in webs of connections. In social-mediated contexts, connections that form among users to create networks can enable or constrain communicators' pursuits of outcomes like public attention. For instance, an organization located centrally in a network may be inherently better positioned to get attention due to its number of ties. However, we recognize communicators are not bound to their network positions; they have the agency to change their positions through communicative actions (Burt, 1992).

In this article, we draw from the strategic communication literature on network management (O'Connor and Shumate, 2018; Sommerfeldt and Yang, 2017; Yang and Taylor, 2015) and social network research in organizational science (Holme, 2005; Rombach et al., 2014) to propose a network contingency model of public attention (Figure 1). The model begins by proposing that network-building strategies (Yang and Taylor, 2015) can situate organizations into "star" and "community" network positions (Saffer et al., 2019) and influence their ability to get attention. Strategies aiming to achieve star positions focus on each individual actor and on how well the actor is positioned in networks (Borgatti, 2006). Strategies aiming at improving community positions focus on actors' participation in communities, similar to the concept of tertius lungens ("or the third who joins") (Obstfeld, 2005). The focus here is in what circles the actor joins, regardless of the actor's own positions. Then the model seeks to explain how the network structures of social-mediated communication influence NGOs' strategic communication choices. 


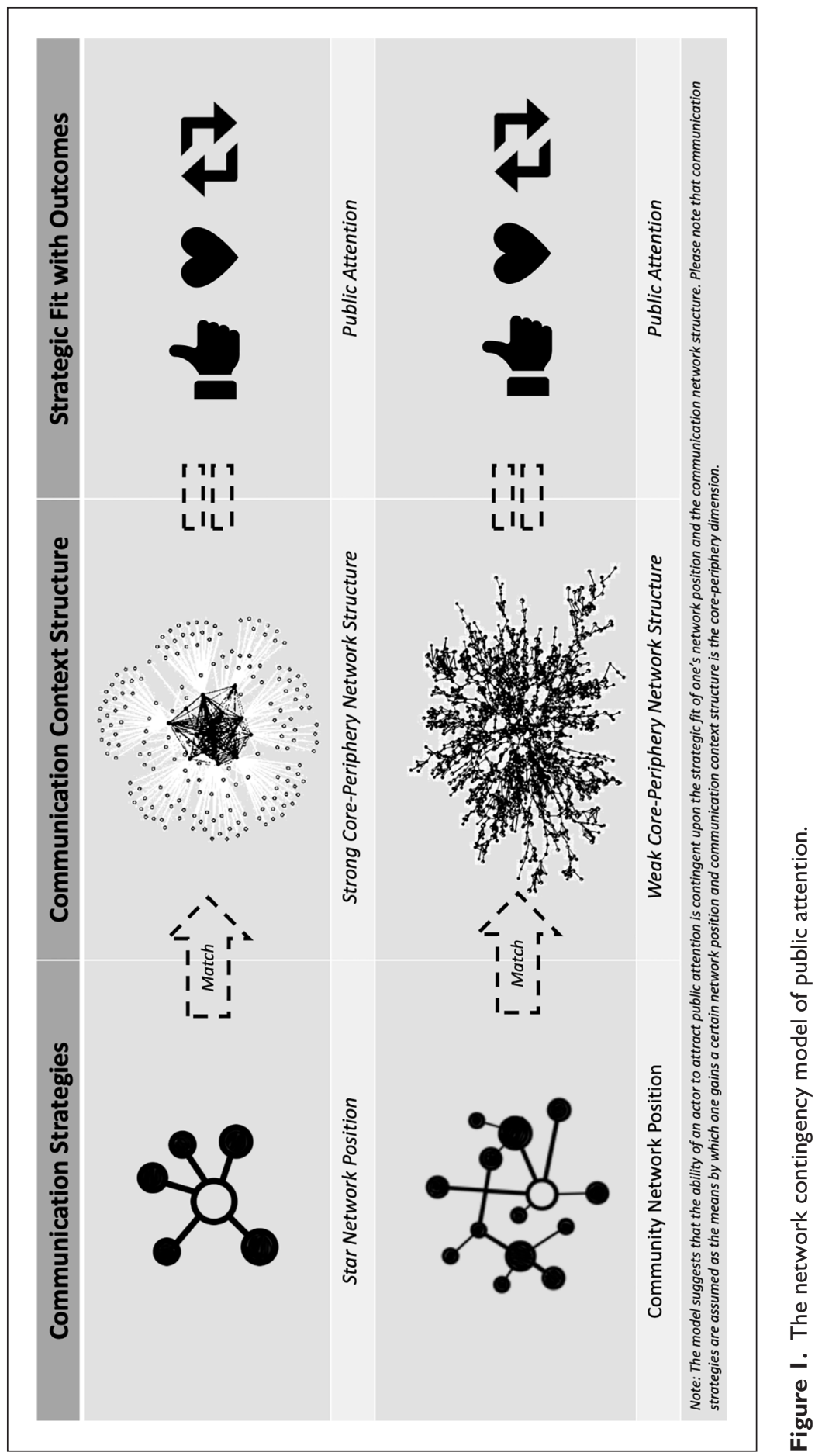


Here we recognize that different communication contexts create network structures that vary by structural features like network density, centralization, and segmentation (Stokman, 2001). One critically important structural feature is the degree to which a network features a strong or weak core-periphery structure (Holme, 2005). With this model, we argue that effective strategic communication needs to identify the fit between relationship-building strategies, network positions, and structural features of the communication context.

Our empirical case for testing the model is drawn from the global refugee crisis. The United Nations Refugee Agency (2018) estimated that 1 in every 110 persons on the planet is a refugee. The ongoing refugee crisis is a pressing challenge, and the political and social stability of countries, basic human rights for millions, and the sustainable development of our planet are threatened. Yet, this refugee crisis is one of many pressing issues competing for public attention. With public attention being a scarce resource as the production and dissemination of information and media content continue to become cheaper, more convenient, and more abundant (Webster, 2014), it is vital for strategic communicators to know how to garner public attention (Galloway, 2017). In the following sections, we first provide an overview of our model and discuss each key concept. To test the model, we combine data mining, social network analysis, and machine-learning techniques to analyze a large-scale Twitter discussion network. The results provide empirical support for the predictive value of our model and offers critical insights to improve NGOs' social media advocacy efforts.

\section{Network contingency model of public attention}

Taking a strategic network management perspective, the network contingency model of public attention assumes that all communicators - organizations and individuals - are situated within webs of relationships that form communication networks. This assumption is especially relevant in the digital space because digital communication mainly takes place in networks (Morales et al., 2014). If we consider relationship-building strategies as a form of strategic communication (O'Connor and Shumate, 2018; Yang and Taylor, 2015), it is valuable to consider how different network structures may call for different relationship-building strategies.

The network contingency model of public attention has three theoretical components. The first component is concerned with individual actors' network positions. Network positions refer to the unique patterns of communicators' connections (Shumate and Dewitt, 2008). A social actor's network position is a microlevel concept (also known as a node-level concept in network science) that demarcates where each actor is located within a network. Actors' patterns of relationship-building strategies can form unique network positions (Shumate and Dewitt, 2008; Yang and Taylor, 2015). Our model assumes that one's network position may present advantages and disadvantages for attracting public attention depending on the network structure of the communication context. For example, an actor with a central network position may be well-positioned to receive public attention from their followers if the network structure of the communication context favors such a position. 
The second component in our model is the network structure of the communication context. Network structures are macrolevel concepts (also known a global-level concepts in network science) that take the aggregated patterns of connections among all members in an entire network. For instance, the density of a network gives an overall indication of a network's connectedness. In a dense network, actors are well connected to one another while in a sparse network actors have few connections to one another. Indeed, other network structural dimensions like network centralization or fragmentation can provide instrumental value (Stokman, 2001); however, we focus on the core-periphery dimension of network structure. This is because the degree to which a network features a strong or weak core-periphery structure provides different contexts for various forms of social influence to propagate through social networks (Bastos et al., 2018; Csermely et al., 2013; Huang and Huang, 2019). A strong core-periphery structure is more hierarchal, with a few actors having the majority or plurality of connections to others in the network. A weak core-periphery structure, on the other hand, is more "horizontal" with a more even distribution of connections among actors and where mobility between the network core and the periphery is more likely. Network positions that allow one actor to achieve salient social influence in a strong core-periphery network may not serve the actor well in a weak core-periphery network.

The last component of our model is the strategic contingency of the previous two components for gaining public attention. Our model suggests that organization's strategic communication strategies should be contingent on their network positions and the level of core-periphery structure of communication contexts. Using one's network position and the network structure, our model predicts that some network positions excel in certain communication contexts like strong core-periphery structures whereas other positions fare better in weak core-periphery structures. Each component is discussed below.

\section{Network positions as outcomes of communication strategy}

Strategic communication scholarship has long considered relationship management as a core function. However, the dominant paradigm is rooted in a dyadic approach to studying relationships and overlooks the complex connections among organizations and stakeholders (O'Connor and Shumate, 2018; Yang and Saffer, 2019). The dyadic approach is increasingly inadequate and outdated in the networked society where relationships and networks are ubiquitous (Iribarren and Moro, 2011).

Yang and Taylor (2015) applied a network approach to re-conceptualize relationship management as a strategic network management. The strategic network management approach posits that strategic communication strategies affect dyadic relationships and could change communicators' network positions and network communities. Building from this approach, Sommerfeldt and Yang (2017) explained that social movement organizations need to adapt their network-building strategies to their goals. For instance, if the organization's goal is to build a coalition to push for legislative changes, it may engage in communication strategies that foster strong ties with key decision makers. Or, if the organization wishes to mobilize a large-scale protest, it may seek to amplify the coalition's messages through engaging in a social media campaign that widely disseminates 
the message. Doing so builds weak ties - connections to others who are otherwise disconnected-to further the coalition's communication goals.

In essence, the strategic network management approach posits that network-building strategies can be strategically planned, empirically measured, and modified according to organizational objectives and resource constraints (Yang and Taylor, 2015). It can also help organizations accomplish communication goals like other relationship management strategies. Recently, Saffer et al. (2019) applied the approach and identified communicators' star-like and community-like network characteristics in Twitter discussion networks to understand how each type of positions influence the public engagement outcomes. As further discussed below, the star and community positions may influence one's ability to attract public attention.

Star network position. The star network position does not refer to one specific network measures; rather, it captures a group of network measures that "focuses specifically on a focal node (i.e. the communicator) and considers how well positioned that node is within a network" (Saffer et al., 2019: 5). In the network literature, an extensive line of research studies the "key player problem" (for review, see Borgatti, 2006). A star network position begins by accounting for the connections a communicator sends or receives (also known as in- and out-degree centrality; Borgatti, 2006). Moving further out from the focal communicator, the connections among a communicator's connections can reveal how advantageously the communicator is connected. For instance, a focal communicator can be positioned between two others who are not already connected. By brokering or filling a structure hole between two otherwise unconnected others, the communicator is advantageously positioned according to Burt's (1992) structural holes theory. In the case of this study, such position may lead to more opportunities to receive public attention. Similarly, effective network size is a metric that captures the degree to which an actor has meaningful, non-redundant connections to others (Burt, 1992). Along with brokerage, effective network size provides a node-level metrics to identify communicators' star positions.

Taken together, possessing star positions allows a communicator to have more connections, connections with powerful others, or more connections with diverse others. A star position can be advantageous for a range of communication goals (Borgatti, 2006).

Community network positions. In networks, actors do not randomly connect with others; rather, the process of social selection creates clusters of small groups, cliques, and subnetworks (Iribarren and Moro, 2011). The small clusters are the microstructures within a larger social network that are the basic building blocks of communities. The members of the same group often share overlapping connections with each other. In these clusters, members can communicate, develop strong ties, and coordinate their communication efforts. We refer to the network measures that describe an actor's membership in different clusters as the "community" network position.

In contrast to the star network position, a community network position considers the groups that a node belongs to (Saffer et al., 2019). The focus is no longer on whether or not one is a star, but whether or not one is a part of well-connected subgroups, or community, within a network. Network research has developed a number of metrics that reveal different types of groups like cliques, triads, and k-cores based on patterns of connections 
(Borgatti, 2006). We argue that although members of these clusters may not all be "stars," their participation in network clusters could shape communication outcomes. For example, Dholakia et al. (2004) found in groups with high "neighborhood solidarity" - tightly bounded, densely knit groups - that consumers' social identities and perceptions of norms significantly differed from groups that are loosely bounded and sparsely knitted.

Our model focuses on the star and community positions for two main reasons. First, in terms of strategic orientation, the two positions represent two ends of a spectrum. The star position is individual-centric. To achieve a star position, actors need to maximize their individual network positions. The community position is collective-centric. To improve community position, actors need to join more "circles" or invite more to join their "circles" (Obstfeld, 2005). Second, as we further discuss below, the star position corresponds with being core in a core/periphery structure. Previous literature tends to consider being star or members of the core as inherently advantageous (Borgatti, 2006). Nevertheless, recent social movement research reveals that both star and community positions offer actors unique power in social influence and mobilization (Barberá et al., 2015). Our model questions previous assumptions about stars and asks if there are conditions in which being star may not be as advantageous as being in the right community, and therefore broadens theoretical considerations and offers new research directions. The key question we explore here is which network position is more valuable under what circumstances. Building from relationship network management approach, Sommerfeldt and Yang (2017) theorized that network-building strategies must be appropriate to communicators' goals and communication contexts. The next section turns the attention to the structural dynamics of communication contexts.

\section{Core-periphery structural dynamics of communication context}

Networks can be described and studied from a range of local or individual, intermediated-scale or meso-scale, and global perspectives (Rombach et al., 2014). One type of important meso-scale network structure is core-periphery structure (Borgatti and Everett, 2000), which refers to the degree to which "one part of the network constitutes a densely connected core that also is central in terms of network distance, and the rest of the network forms a periphery" (Holme, 2005: 1). Members of the core are deeply entrenched in their social systems and therefore tend to closely share ideas and habits. Members of the periphery are tied to the rest of the network by looser linkages and are scarcely connected to each other.

Core-periphery structure is important in our consideration of the network contingency model because of (1) its pervasiveness, (2) its influence on actors' communication outcomes, and (3) its unequal distribution on online platforms and communication contexts. Each of these considerations is further discussed below.

Pervasiveness. Many social, economic, technological, and biological networks exhibit a core-periphery structure (Csermely et al., 2013). The structure is widely observable in gene regulatory networks, neuronal cell networks, ecosystems, animal and human networks, power-grids or transportation networks, Internet-of-things, and even world system (Csermely et al., 2013). For example, Wallerstein (2011) argues that nation-states in 
the world are connected by economic, political, and technological relationships and form a world system. The world system network exhibits a clear core-periphery structure where some nations are core nations, whereas others locate in periphery positions. Such a system determines global economy, diplomacy, telecommunication, and even news flows (Barnett, 2001; Kim and Barnett, 1996). Similarly, using Twitter data, Yang et al. (2018) showed that core-periphery is a common network structure that is widely present in online communities. Their analysis suggested that once communities are detected, core-periphery analysis can reveal the influential (core) users in every community.

Influence on communication outcome. The idea that core-periphery structures affect actors' performance and communication outcomes has also enjoyed considerable attention in the social network literature (Borgatti and Everett, 2000). Research has examined how coreperiphery positions influence individuals' creative performance (Cattani and Ferriani, 2008) and how core-periphery networks affect networked framing processes (Bennett et al., 2018). Huang and Huang (2019) found that in the epidemic spreading process, members of the core have a higher likelihood to be influential spreaders. In the context of social-mediated protests, Jost et al. (2018) found that core-periphery dynamics are especially important in the mobilization for collective actions. Although core participants demonstrate more intense Twitter use than periphery users, the success of those movements to mobilize large number of citizens often depend on the degree to which activists can activate the critical periphery users, who account for the majority of participants.

Unequal distribution. Given the important influence of core-periphery structure on communication outcomes, it is necessary to also recognize that not all social networks have the same level of core-periphery structure. For instance, Bastos et al. (2018) found that communities where specialized information is shared tend to feature a high level of coreperiphery structure. By contrast, in communities where general information is discussed, their network adopts a decentralized structure that features a low level of core-periphery structure . As noted by Csermely et al. (2013), the "absolute and relative size of the core is a key property of core-periphery structures." Csermely and colleagues further noted that an extensive core "allows a larger flexibility and adaptability of the network"; whereas an exclusive core facilitates control of flows in networks (p. 95).

The differences between strong and weak core-periphery network structures have important implications that must be considered (Holme, 2005). In strong core-periphery networks, the core is small and only a relatively handful core actors receive a majority of incoming/outgoing ties. In other words, only a small percentage of actors can enjoy particularly high-degree centrality (Rombach et al., 2014). In weak core-periphery networks, the difference between members of core and periphery is less distinctive and a much larger section of network members are part of the core. In other words, there is a higher opportunity for members of the network to obtain core membership.

Core-periphery structure arises through network degree assortativity, which refers to "the degree to which nodes with similar degrees connect to each other" (Ahuja et al., 2012: 437). Positive assortativity, which refers to the condition where high-degree nodes connect to other high-degree nodes and low-degree nodes associate with other low-degree nodes, lead to strong core-periphery structure. Negative assortativity occurs 
when high-degree nodes refer to interact with low-degree nodes, which lead to low core-periphery structure (Ahuja et al., 2012).

Drawing from the work of Saxton and Waters (2014), we argue that on social-mediated platforms, actor-motivated communication is more likely to lead to strong core-periphery structure, whereas message-motivated communication is more prone to weak core-periphery structure. In actor-motivated communication, a communicator initiates communication to engage with specific actors, whereas in message-motivated communication, a communicator initiates communication to engage with the content or message. Following this logic, on Twitter, mention and retweet networks are likely to be substantially different in network structures. Saxton and Waters define mentions as an indicator of actor-motivated communication and retweet as an indicator of message-motivated engagement. The act of mentioning someone normally occurs when one user wants to get another user's attention. Accounts associated with high prestige, visibility, or relevance are far more likely to be mentioned. The motivation that drives tie formation in a mention network is likely to favor elites, and not every Twitter user could achieve such a status. Therefore, in this study, we propose that actor-motivated communication networks are more likely to feature strong core-periphery context.

Meanwhile, when users bookmark or retweet a tweet, they could do so because of the content of the tweet interesting. Since any user could potentially come up with an interesting tweet, the difference between influencers and regular users may not be as pronounced in a retweet network. Although it is likely that some of the motivations do overlap, in a large-scale network where millions of people mention and retweet, the overall networks may assume different overall structural features. Our model thus predicts the following hypothesis:

H1: The mention network features a strong core-periphery structure, while the retweet network features a weak core-periphery structure.

\section{Contingency between communication strategies and communication context}

The last component of the network contingency model of public attention is the concept of strategic contingency, which refers to the idea that actors' communication outcomes in a networked communication context are contingent upon the fit between actors' network positions and the network structure. In this study we draw upon the literature on information diffusion in networks (Iribarren and Moro, 2011; Morales et al., 2014; Spitzberg, 2014) and propose two sets of conditions that would lead to strategic fit.

Condition I: star network position for strong core-periphery network structure. The information diffusion research has documented that on platforms such as Twitter, some users are able to influence others and get their messages spread through networks (Morales et al., 2014). The key to success when propagating information depends on a number of factors such as the popularity of the users, posting frequency, and the novelty and resonance of message content (Spitzberg, 2014). In addition, research showed that information propagation models vary in networks with different structures (Iribarren and Moro, 2011). 
A defining feature of strong core-periphery context is the small core that consists of a handful of key members who have developed dense connections between themselves. Elites enjoy disproportionate level of influence in this context. In this type of context, the most efficient approach to attract public attention or to disseminate information, when everything else equal, is through working with elites in the small core. Members of the core enjoy the lion's share of resources and connections, and the voice of one elite influencer may surpass that of many periphery members. Therefore, we propose that in a strong core-periphery structure, star network positions are most important in shaping actors' ability to attract public attention.

$\mathrm{H} 2$ : In a network with a strong core-periphery structure, star network positions are more important factors that shape actors' influence on public attention than community network positions.

It is worth noting that public attention is measured differently across multimedia contexts (Bennett et al., 2018). We focus on Twitter and measure public attention based on the number of mentions (for mention networks) and the total number of retweets (for retweet networks) (Guo and Saxton, 2018).

Condition II: community network positions for weak core-periphery network structures. By contrast, a weak core-periphery structure has a less pronounced difference between core and periphery actors (Holme, 2005). The structure is more decentralized and may have multiple centers. Although members of the core still enjoy considerable advantages, periphery members enjoy considerable opportunities to be exposed to different sources of information (Cattani and Ferriani, 2008). Information propagation in this type of context does not necessarily follow a power-law pattern. In fact, Watts and Dodds (2007) used a series of computer simulations and found that in this type of relatively "flat" context, large cascades of information diffusion are not driven by influential but by a critical mass of easily influenced individuals. In other words, in contexts with weak coreperiphery structure, working with elite influencers alone may not give actors much advantages. Instead, actors' voices can be amplified through memberships in network clusters that provide close access to others. We propose that in a weak core-periphery structure, community network positions are far more consequential on an actor's ability to attract public attention. Hence, we propose the following hypothesis:

H3: In a network with weak core-periphery structure, community network positions are more important in shaping actors' influence on public attention than star network positions.

\section{Materials and methods}

\section{Sampling procedures}

We first reviewed the refugee crisis media coverage and developed a search string to query the major world publications in LexisNexis. Relevant news articles were gathered 
in two ways. First, a research assistant queried LexisNexis and gathered the number of articles published each day in $2016 .{ }^{1}$ Then we identified 26 news spikes, which are periods in 2016 when the amount of media coverage mentioning refugees was two standard deviations above average. Two news spikes with similar amounts of coverage were selected as the window for gathering tweets. The first news spike occurred between April 18 and 19, and the second news spike occurred between August 29 and 31. Data from the first news spike were used for data analysis and model training, and data gathered from the second were used for model testing and verification.

Once we determined the window of time, we used DiscoverText (Shulman, 2011) to purchase tweets about the refugee crisis during the two news spikes. ${ }^{2}$ After an initial review of the media coverage and tweets, we purchased all public tweets that mentioned "refugee" or "illegal immigrants." We used these broad search terms to capture the full spectrum of tweets. The Twitter data included users' bios, number of followers and friends, and total number of tweets. In addition, the data also included the number of retweets and a list of users mentioned in/retweet a tweet. We constructed the mention and retweet networks based on users' mentioning or retweeting relationships. Both networks were further imported into ORA, a "network analysis toolkit for graphical, statistical and visual analytics on both social network and high dimensional networks that vary by time and/or space" (Carley, 2014: 1219). The network analysis returned network metrics for all users, not just NGOs, included in the dataset.

\section{Operationalization}

Identifying NGOs. In order to identify the NGOs in each news spike, we first queried all users' Twitter handles and bio-data to identify accounts self-disclosed as NGOs/nonprofits. Then a team of research assistants reviewed each account's bio and searched online to verify whether each account was associated with an NGO. In total, we identified 213 NGOs in the first news spike and 91 NGOs in the second.

Dependent variables. Informed by previous research (Guo and Saxton, 2018), we operationalized public attention using two dependent variables: the number of mentions and the total number of retweets. The number of mentions refers to the total number of times an NGO's Twitter handle was mentioned by others during a news spike. Following Guo and Saxton (2018), we defined the number of retweets as "the total number of retweets of an organization's tweets by other users" during a news spike (p. 14).

Independent variables. We operationalized 20 independent variables, ${ }^{3}$ and we introduce these variables in three types: account characteristics, star network positions, and community network positions (Table 1).

Twitter account characteristics. The first set of variables capture the standard characteristics of Twitter accounts. The total number of followers indicates the number of accounts that follow each Twitter account. The total number of friends denotes how many other Twitter accounts one account follows. The total number of tweets assess how frequently one account tweeted during a news spike period. 
Table I. The mean $(M)$ and standard deviation $(S D)$ for all independent variables.

\begin{tabular}{|c|c|c|c|c|}
\hline \multirow{2}{*}{ Variables } & \multicolumn{2}{|c|}{ Mention network } & \multicolumn{2}{|c|}{ Retweet network } \\
\hline & $M$ & $S D$ & $M$ & $S D$ \\
\hline \multicolumn{5}{|l|}{ Account status } \\
\hline \# of followers & & $20,532.9$ & $3,23,182.6$ & 9701.3 \\
\hline \# of friends & & 1227.8 & 7671.0 & $153||$. \\
\hline \multicolumn{5}{|l|}{ Use pattern } \\
\hline \# of tweets & & 3.8 & 93.7 & 1.7 \\
\hline \multicolumn{5}{|l|}{ Centrality measures } \\
\hline Authority centrality & & 0.0 & 0.1 & 0.0 \\
\hline Eigenvector centrality & 0.0 & 0.0 & 0.0 & $1,93,664.4$ \\
\hline Hub centrality & 0.0 & 0.0 & 0.0 & 6530.6 \\
\hline In-degree centrality & 0.0 & 0.0 & 0.0 & 3.2 \\
\hline Out-degree centrality & 0.0 & 0.0 & 0.0 & 0.0 \\
\hline Total degree centrality & 0.0 & 0.0 & 0.0 & 0.0 \\
\hline \multicolumn{5}{|l|}{ Structural hole measures } \\
\hline Brokerage & 0.9 & 0.2 & 0.9 & 0.0 \\
\hline Structural holes constraint & 0.8 & 0.3 & & 0.0 \\
\hline Structural hole effectiveness & 1.9 & 8.5 & & 0.0 \\
\hline Structural hole efficiency & 1.0 & 0.1 & & 0.0 \\
\hline \multicolumn{5}{|l|}{ Neighborhood effect measures } \\
\hline Average neighbor in-degree & 0.2 & 0.9 & 0.3 & 0.3 \\
\hline Average neighbor out-degree & 0.2 & 0.9 & 1.5 & \\
\hline Average neighbor total degree & 62.9 & 187.8 & 515.3 & \\
\hline \multicolumn{5}{|l|}{ Clustering pattern measures } \\
\hline Triad count & 0.1 & 0.4 & & \\
\hline Clique count & 0.1 & 0.6 & & 4.4 \\
\hline Clustering coefficient & 0.1 & 0.2 & & 3.3 \\
\hline K-core & 0.0 & 0.0 & & 1267.2 \\
\hline
\end{tabular}

SD: standard deviation.

Star network positions. Accounts' star network positions are assessed by two sets of measures. The first set focuses on how well an account is connected, and includes six indicators: authority centrality (the extent to which a node's incoming ties are from others with many outgoing ties), eigenvector centrality (the extent to which a node connects with other well-connected nodes), hub centrality (the extent to which a node's outgoing ties are to nodes with many incoming ties), in-degree centrality (the extent to which a node has many incoming ties), out-degree centrality (the extent to which a node has many outgoing ties), and total degree centrality (the combined score of in- and out-degree centrality).

The second set are measures based on Burt's (1992) structural hole theory, which measures the likelihood for an account to be brokers in relationships. The second set has a total of four indicators: brokerage (the degree to which a node can act as a broker between its direct connections), structural holes constraint (the degree to which a node is 
constrained from acting because of its ties to others), structural hole effectiveness (the effective size of a node's ego network based on redundancy of ties), and structural hole efficiency (the fraction of nodes in a node's ego network that are not redundant).

Community network positions. Two sets of measures were used to capture accounts' community network positions. The first set focuses on surveying the neighborhood in which accounts are embedded in. These measures are average neighbor in-degree, average neighbor out-degree, and average neighbor total degree. The second set focuses on accounts' memberships in different clusters. Four metrics were used: (1) clique count denotes the number of distinct cliques to which each NGO belongs, ${ }^{4}$ (2) triad count reports the number of triads a node belongs to $(\mathrm{A} \rightarrow \mathrm{B}, \mathrm{B} \rightarrow \mathrm{C}, \mathrm{C} \rightarrow \mathrm{A})$; (3) k-cores metric "identifies groups in a network by recursively removing all nodes with fewer than $\mathrm{k}$ links (thus 0-core includes all nodes in a network while 2-core includes only nodes with two or more links)" (Altman et al., 2018: 300); and (4) the clustering coefficient (the density of a node's ego network).

\section{Data analysis}

Social network analysis (SNA). Two types of networks - mention and retweets - were analyzed. To reveal the network positions of all involved users, standard network procedures were performed to calculate all users' network positions (Borgatti, 2006). The network metrics described in the previous section were analyzed using ORA (Carley, 2014).

Machine learning and regression tree. Machine learning is an interdisciplinary field of research with a wide variety of applications (Jordan and Mitchell, 2015). Among different machine-learning methods, regression tree belongs to a family of methods that apply supervised classification and decision tree methods to identify critical factors and thresholds in large-scale data (Breiman, 2017). Regression tree combines the strength of decision trees with the ability to model numeric data. Regression tree makes predictions based on the average values of example cases that reach a leaf. At each leaf, a multiple linear regression model is built from the examples reaching that node (Lantz, 2013). This type of method uses automatic feature selection and does not require users to specify the model in advance and therefore can reveal unknown patterns in large-scale data. Moreover, the approach does not require similar assumptions such as none-interdependency among observations like linear regression. As such, this type of method can be especially useful for large-scale data involving social network measures (Lantz, 2013).

\section{Results}

\section{Descriptive statistics}

During the first news spike, there are 21,500 tweets in the mention network. The mention network contains a total of 67,361 unique users that are connected through 27,339 mention ties (Density $=0.0006$ ). The retweet network, with 148,648 tweets, contains a total of 103,816 unique users that are connected through 103,816 retweet ties 
$($ Density $=0.00014)$. Users' geographic locations suggest that this event has captivated worldwide attention. Users from around the world participated, with users from United States, United Kingdom, and Australia leading the way.

\section{Differences in mention and retweet networks}

Hypothesis 1 predicts that the mention network features a strong core-periphery structure, while the retweet network features a weak core-periphery structure. To test this hypothesis, a couple of steps were taken. First, we calculated the basic network descriptive statistics for both mention and retweet networks and ran a core-periphery analysis (Holme, 2005). The analysis shows that the mention network contains 67,361 nodes, with 3865 members below to a core group. There are 2869 dyads and 795 triads in this network. This structure suggests considerable interactions in this network and that a clear core-periphery structure is present, with only a small number of members holding core positions (5.74\%). The retweet network contains 103,816 nodes, with 35,831 members below to a core group. There are a total of 1994 dyads and 739 triads in this network. This is a relatively flat network, both at the local and at the overall level, with very few triads and many members being part of the core group $(34.51 \%)$.

In short, while the size of the retweet network is only two times larger than the mention network, the core in the retweet network is ten times bigger than the core in the mention network. With this evidence, $\mathrm{H} 1$ is supported. Mention network reflect strong core-periphery structure, whereas retweet network reflected weak core-periphery structure. This important distinction provides foundation for further analysis and hypotheses testing.

\section{Testing the network contingency model of public attention}

Hypotheses 2 and 3 predict that star network positions allow actors to emerge as influencers in a strong core-periphery structure network, whereas community network positions allow actors to excel in a weak core-periphery structure network. To test these hypotheses, training models were built on the first dataset to build regression tree models that best explain the outcome. The tree models were further used to predict outcomes in the second dataset to see how well the model holds in the face of new data (Breiman, 2017). In this section, we again report findings separately per the mention and retweet networks.

Mention network. This training model ran a regression tree on a total of 21,500 cases. The decision tree model achieved a good model fit, with a mean square error (MSE) of 0.093, which means the difference between the model's predictions and the true observed score is only about 0.093 . On a transformed scale that range from 0 to 3.5 , the difference is small and suggests an excellent fit. The model used frequency of mention as the dependent variable and all 15 previously discussed independent variables. Once the regression tree method identified a plausible model, we further tested the validity of this model with Twitter data from the second news spike. The model was able to reliably predict mention frequency in the second event. The correlation between predicted value and actual value is .67 , and MAE is 0.82 , exceeding the recommended value of 0.50 . 
Each variable's importance level differs at different decision points, and the model also computed the overall importance of each variable. Figure 2(a) shows the decision tree, and Table 2 provides details on each major decision point. Because average neighbor indegree was used first in the tree, it is the most important predictor of users' frequency of mention. In this dataset, there are 18,312 cases with average neighbor in-degree smaller than 0.01 and 3188 cases equal or larger than 0.01 . In addition, nodes indicated by * are terminal or lead nodes in the decision tree, which means that the results are statistically significant. Other variables that significantly and positively affect mention frequency are structural hole effective network size, in-degree centrality, and authority centrality. Structural hole constraint significantly and negatively affects mention frequency. Considering that average neighbor in-degree is a community indicator, but all other significant indicators are star network positions, we conclude that $\mathrm{H} 2$ was supported.

Retweet network. Similar procedures were taken to test the effect of variables in the retweet network. The training model ran regression tree on a total of 63,269 cases. The decision tree model achieved a good model fit, with a mean square error (MSE) of 0.089, which means the difference between the model's predictions and the true observed score is only about 0.089 . On a transformed scale that range from 0 to 5.2 , the difference is very small and suggests that the model is an excellent fit. Once the regression tree method built the model, we further tested the validity of the model with Twitter data from the second news spike. The model was able to reliably predict mention frequency in the second event. The predicted mean is 1.41 and the actual observed mean is 1.58 . The correlation between predicted value and actual value is .67 , and MAE is 0.82 , far exceeding the recommended value of 0.50 .

The model used the number of retweets as the dependent variable and all 15 previously discussed independent variables. Each variable's importance level differs at different decision points and the model also computed the overall importance of each variable. There are two variables that overwhelmingly dominate the importance rank: average neighbor total degree (46) and number of tweets (21). Figure 2(b) shows the decision tree, and Table 3 provides details on each major decision point. Because average neighbor total degree was used first in the tree, it is the single most important predictor of accounts' frequency of mention. As given in Table 3, when cases meet average neighbor total degree $\geqslant 34.05$ (there are 37,365 cases that meet this threshold), if they sent out more than one tweet, they would be guaranteed with a higher level of influence $(\geqslant 1.49)$ than the mean $($ mean $=1.43)$. The analysis essentially confirmed that community indicators dominated actors' chance to emerge as influential in the retweet network, and H3 was supported.

\section{Discussion}

In the study we proposed a network contingency model of public attention. This model recognizes that the public's attention is sought after by many in expansive networks where various actors are competing for the public's attention. The model extends the strategic network management approach (O'Connor and Shumate, 2018; Sommerfeldt and Yang, 2017; Yang and Taylor, 2015), and argues that the strategic fit between 


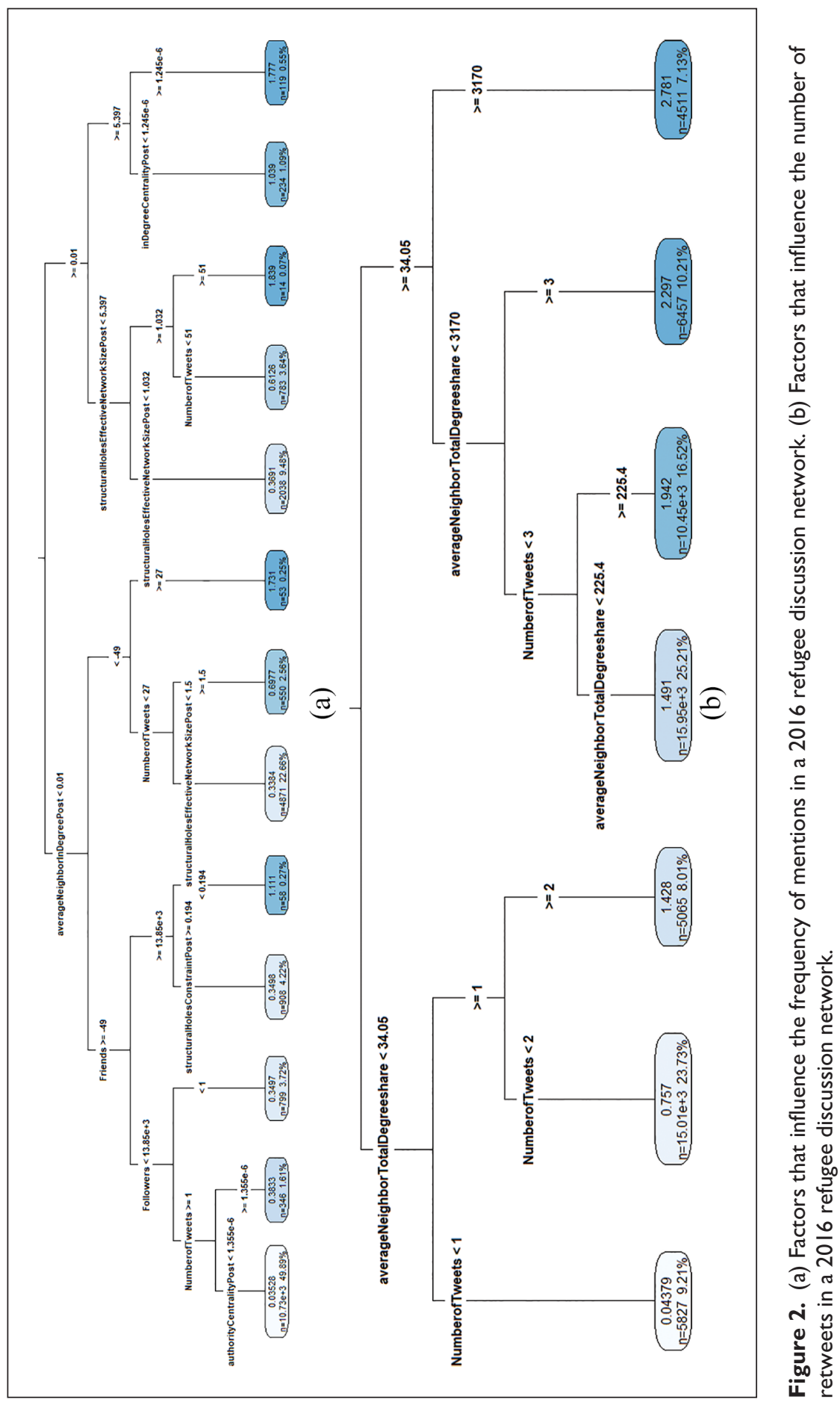




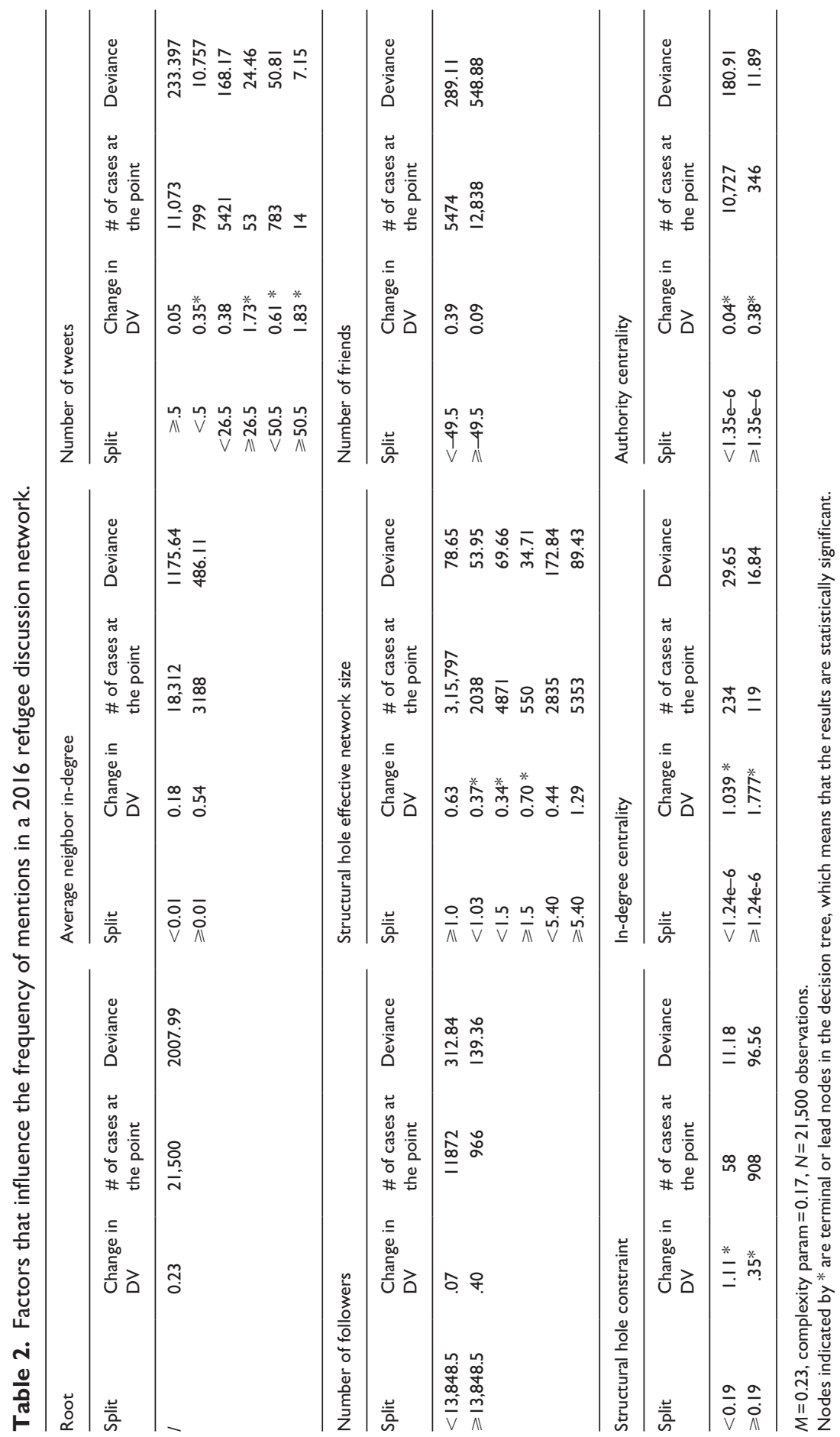




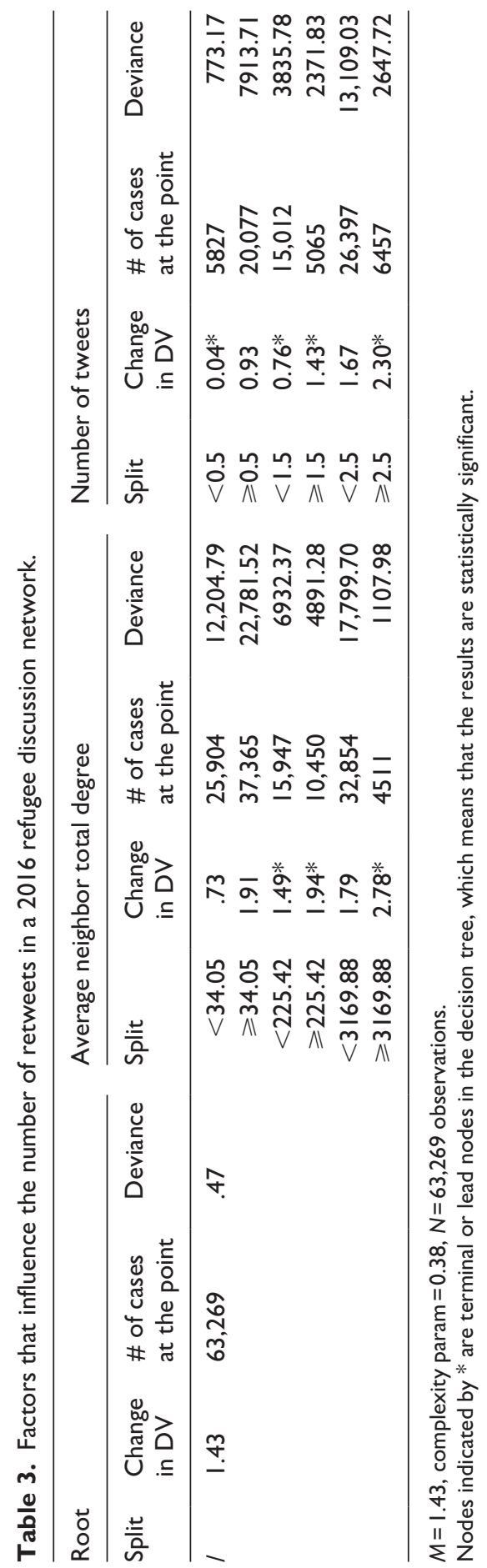


relationship-building strategies and communication contexts significantly improves communicators' influence in social media discourses. Empirical evidence from this study provides strong support for the model. Below we further expand on the theoretical, methodological, and practical implications.

\section{Theory building and the network contingency model of public attention}

The current era is characterized by drastically changing social, political, and technological realities. Strategic communicators need to adapt to these changes and embrace multidisciplinary research to advance their practices. The network contingency model of public attention reflects valuable explorations in this direction. The model draws upon several subfields in communication, marketing, and network research, and recognizes the contingency between relationship network-building strategies and specific communication contexts. The model recognizes that public attention is and inherently interdependent phenomenon. One's ability to attract public attention is not done in isolation; rather, it occurs through and within networks of connections. Our study presents strong evidence for how various network elements interact in ways that have implications on communicator's ability to capture attention. Several important theoretical implications emerge from the analysis that warrants further attention.

First, the verification of hypothesis 1 supports the idea that some communication contexts feature strong core-periphery contexts, whereas others show weak core-periphery contexts (Holme, 2005). Communication scholars have recognized the importance of core-periphery structures in social networks, yet much of that work been applied limited to world system studies (Barnett, 2001; Kim and Barnett, 1996). Rarely have studies differentiated the levels of core-periphery or investigated how such difference may affect communication outcomes until now.

The results from assessing the network contingency model of public attention demonstrate the importance of factoring in the core-periphery structure of the communication contexts. Our model recognizes that communication contexts may differ in their levels of core-periphery structure in ways that influences what types of communicators are better positioned to capture public attention. It is important to note that the concept of coreperiphery has implications beyond social-mediated communication. Since core-periphery structure is pervasive in all sorts of networks, it is likely that the level of core-periphery structure may also influence communication outcomes in group communication and organization communication, to name a few examples. Much of the work on communication networks considers either actors' network positions or network structure. Our model provides a holistic framework to consider actors' network positions and the network structures of the communication contexts. Such an approach allowed us to reveal the importance of strategic fit.

Second, findings support predictions of the model and reveal the importance of strategic fit. The analysis showed that in the context with strong core-periphery structure, actors' star network positions played dominating roles in shaping actors' chance to be influential. In the context with weak core-periphery structure, communicators' community network positions such as total neighborhood degree were most influential. It is necessary to mention that the machine-learning models also took into consideration of users' 
characteristics such as their number of followers and the frequency of tweets. These models supported the importance of network positions in shaping actors' chance to be influential while taking all variables into account. It is likely that in a large and globally sparse network such as the global discourse network on refugee issues, many actors could have large follower bases or are frequent Twitter users. However, if they do not have advantageous network positions appropriate to the communication contexts, regardless of their characteristics, it would be difficult for them to emerge as influencers.

\section{Methodological implications for studying public attention}

The new theoretical framework also calls for innovative research methods. In this study we blend together data mining, SNA, text mining and machine learning. These big data analytics are innate to the digital communication contexts. The communication industry has started embracing big data analytics with many agencies, corporations, and organization routinely incorporate big data analytics in strategic campaigns and decision-making. Communication theories and methodologies should also embrace and reflect the current trends.

In this study, we demonstrate that methods such as SNA and machine-learning models are compatible. Machine-learning models can accommodate complex, interdependent data. As our communication networks grow in complexity and sizes, the combination of machine learning and network analysis can be a fruitful forefront for future research. Moreover, the triangulate combination of network analysis and text-mining methods could reveal both structural and semantic aspect of digital communication. Future research should further explore how computational methods could help uncover hidden structural patterns that are influential in complex communication phenomena.

\section{Applied implications for garnering public attention}

The proposed model offers guideline for advancing strategic communicators' social media campaigns. Nowadays, resourceful strategic communicators such as organizations are increasingly integrating big data metrics into their social media strategies. Often these metrics focus on elements like the sentiment of tweets or number of followers. Those existing metrics may be misleading in the sense that they place the focus entirely on communicators while disregarding the larger communication networks in which their communicative actions take place. Some advanced social media tracking software or companies have features to track basic network characteristics of individuals. However, to the best of our knowledge, most of those software/companies essentially only focus on the "star" type of users to identify influencers. As our analysis showed, that is simply one part of the story. Informed by our model and results, we believe strategic communicators should consider integrating network analytics to understand the structure of the communication environments. In addition, network metrics should incorporate both star and community characteristics. Insights from analytics that incorporated these network metrics could allow strategic communicators like NGOs to develop adaptive strategies for capturing public attention given the network structure of the conversation. 
At the same time, this model also offers insights for efforts to effectively remove or combat communicators with malicious intentions. For example, during the COVID-19 global pandemic, some communicators intentionally spread misinformation that strike fear or even panic. Platform operators or policy-makers could identify malicious influencers based on the type of communication context in which they operate and based on their network positions, and effectively target them to reduce the impact of misinformation that they spread.

\section{Limitations and future research}

This study comes with some limitations that can be improved through further research. First, we operationalized communicators' network positions and the network structures of the communication contexts based on data from Twitter. Inherently, this limited us to only test the model on a single platform. Communicators use a variety of social media platforms to build relationships and accomplish strategic objectives, and need to capture public attention on those platforms too. Despite our focus on Twitter, we believe most of the conceptual elements of the network contingency model of public attention are transferable to other platforms. Future studies could examine if outcomes proposed in the model also appear on other platforms.

Second, our operationalization of ties among communicators did not capture the full range of types of ties (Ihm, 2019). One way to advance this model would be to consider how multiplex networks based on different types of ties (e.g. representational, affinity, etc.) may strengthen or weaken the influence of the network structures of the communication contexts.

Third, our data were derived from the public tweets about a single critical issue in 2016: the global refugee crisis. Future studies could test the model across multiple topics of discussion. In different issue contexts, patterns of discourse may differ. For instance, communicators in an issue space like the refugee issue may have had more time to learn about the issue and developed strong opinions and positions on the issue. Yet, when issues arise with little precedent such as the COVID-19 pandemic, few prior relationships or knowledge may have existed. Publics need to acquire new information and forge new relationships as the issue unfolds. It would be interesting to compare how prior knowledge on issues and the novelty associated with issues may affect variables identified in the current model. Characteristics associated with social issues may be another dimension of variables to strengthen the proposed model.

\section{Conclusion}

The aim of our study was to develop and test a model for understanding how the interplay between communicators' positions and the structure of the networks influences communicators' abilities to garner public attention. We argue that gaining influence and attracting the limited supply of the public's attention depends on a number of network elements. The conceptual model and empirical evidence presented in this study shows that networks can be managed through strategic planning to accomplish strategic communication goals. Overall, with continued research to identify issue-related variables 
that may interact with communicators' positions and communication context, this network contingency model of public attention could provide a much needed theoretical framework to guide future research on public discourse and public attention on a wide range of new media platforms.

\section{Author's note}

Adam J Saffer is now affiliated with University of Minnesota-Twin Cities, USA.

\section{Funding}

The author(s) received no financial support for the research, authorship, and/or publication of this article.

\section{ORCID iDs}

Aimei Yang (iD https://orcid.org/0000-0003-3756-7812

Adam J Saffer (iD https://orcid.org/0000-0001-8032-4256

\section{Notes}

1. LexisNexis only returns a max of approximately 1000 articles per search; thus, it was not possible to search by month or week.

2. Assuming Twitter discussion could influence the media coverage and vice versa, we gathered tweets one day before a news spike to captures tweets prior to news production and gathered tweets a day after to capture tweets that followed the media coverage.

3. Unscaled network outputs were used given that we are comparing two networks with varying sizes.

4. The ORA user manual explains that a "clique is defined as a group of three or more nodes that are all connected together and that cannot be made larger by adding another node" (p. 1121).

\section{References}

Ahuja G, Soda G and Zaheer A (2012) The genesis and dynamics of organizational networks. Organization Science 23(2): 434-448.

Altman N, Carley KM and Reminga J (2018) ORA user's guide 2018. Available at: http://www. casos.cs.cmu.edu/projects/ora/CMU-ISR-18-103.pdf

Barberá P, Wang N, Bonneau R, et al. (2015) The critical periphery in the growth of social protests. PLoS ONE 10(11): e0143611.

Barnett GA (2001) A longitudinal analysis of the international telecommunication network, 19781996. American Behavioral Scientist 44(10): 1638-1655.

Bastos M, Piccardi C, Levy M, et al. (2018) Core-periphery or decentralized? Topological shifts of specialized information on Twitter. Social Networks 52: 282-293.

Bennett WL, Segerberg A and Yang Y (2018) The strength of peripheral networks: negotiating attention and meaning in complex media ecologies. Journal of Communication 68(4): 659-684.

Borgatti SP (2006) Identifying sets of key players in a social network. Computational \& Mathematical Organization Theory 12(1): 21-34.

Borgatti SP and Everett MG (2000) Models of core/periphery structures. Social Networks 21(4): 375-395. 
Breiman L (2017) Classification and Regression Trees. Abingdon: Routledge.

Burt RS (1992) Structural Holes: The Social Structure of Competition. Boston, MA: Harvard University Press.

Carley KM (2014) ORA: a toolkit for dynamic network analysis and visualization. In: Alhajj R and Rokne J (eds) Encyclopedia of Social Network Analysis and Mining. New York: SpringerVerlag, pp. 1219-1228.

Cate F (2002) The CNN Effect is far from clear cut. Humanitarian Affairs Review 5: 4-7.

Cattani G and Ferriani S (2008) A core/periphery perspective on individual creative performance: social networks and cinematic achievements in the Hollywood film industry. Organization Science 19(6): 824-844.

Csermely P, London A, Wu LY, et al. (2013) Structure and dynamics of core/periphery networks. Journal of Complex Networks 1(2): 93-123.

Dholakia UM, Bagozzi RP and Pearo LK (2004) A social influence model of consumer participation in network-and small-group-based virtual communities. International Journal of Research in Marketing 21(3): 241-263.

Galloway C (2017) Blink and they're gone: PR and the battle for attention. Public Relations Review 43(5): 969-977.

Griffith E (2015) Brands are using social media more than ever, and users are ignoring them more than ever. Fortune, 25 August. Available at: http://fortune.com/2015/08/25/social-mediabrands-ignore/

Guo C and Saxton GD (2018) Speaking and being heard: how nonprofit advocacy organizations gain attention on social media. Nonprofit and Voluntary Sector Quarterly 47(1): 5-26.

Holme P (2005) Core-periphery organization of complex networks. Physical Review 72(4): 046111.

Huang JY and Huang JX (2019) Epidemic behaviors in weighted networks with core-periphery structure. Physica A: Statistical Mechanics and Its Applications 528: 121452.

Ihm J (2019) Communicating without nonprofit organizations on nonprofits' social media: stakeholders' autonomous networks and three types of organizational ties. New Media and Society 21(11-12): 2648-2670.

Iribarren JL and Moro E (2011) Affinity paths and information diffusion in social networks. Social Networks 33(2): 134-142.

Jordan MI and Mitchell TM (2015) Machine learning: trends, perspectives, and prospects. Science 349(6245): 255-260.

Jost JT, Barberá P, Bonneau R, et al. (2018) How social media facilitates political protest: information, motivation, and social networks. Political Psychology 39: 85-118.

Kim K and Barnett GA (1996) The determinants of international news flow: a network analysis. Communication Research 23(3): 323-352.

Lantz B (2013) Machine Learning with R. Birmingham: Packt Publishing.

Morales AJ, Borondo J, Losada JC, et al. (2014) Efficiency of human activity on information spreading on Twitter. Social Networks 39: 1-11.

Obstfeld D (2005) Social networks, the tertius iungens orientation, and involvement in innovation. Administrative Science Quarterly 50(1): 100-130.

O'Connor A and Shumate M (2018) A multidimensional network approach to strategic communication. International Journal of Strategic Communication 12(4): 399-416.

Rombach MP, Porter MA, Fowler JH, et al. (2014) Core-periphery structure in networks. SIAM Journal on Applied Mathematics 74(1): 167-190.

Saffer AJ, Yang A, Morehouse J, et al. (2019) It takes a village: a social network approach to NGOs' international public engagement. American Behavioral Scientist 63(12): 1708-1727. 
Saxton GD and Waters RD (2014) What do stakeholders like on Facebook? Examining public reactions to nonprofit organizations' informational, promotional, and community-building messages. Journal of Public Relations Research 26(3): 280-299.

Shulman S (2011) DiscoverText: software training to unlock the power of text. In: Proceedings of the 12th annual international digital government research conference: digital government innovation in challenging times, College Park, MD, 12-15 June. New York: ACM.

Shumate M and Dewitt L (2008) The north/south divide in NGO hyperlink networks. Journal of Computer-Mediated Communication 13(2): 405-428.

Shumate M, Fulk J and Monge P (2005) Predictors of the international HIV-AIDS INGO network over time. Human Communication Research 31(4): 482-510.

Sommerfeldt EJ and Yang A (2017) Relationship networks as strategic issues management: an issue-stage framework of social movement organization network strategies. Public Relations Review 43(4): 829-839.

Spitzberg BH (2014) Toward a model of meme diffusion (M3D). Communication Theory 24(3): 311-339.

Stokman FN (2001) Networks: social. In: Smelser NJ and Baltes PB (eds) International Encyclopedia of Social and Behavioral Sciences. New York: Pergamon, pp. 10509-10514.

United Nations Refugee Agency (2018) Forced displacement above 68m in 2017, new global deal on refugees critical. 19 June. Available at: http://www.unhcr.org/en-us/news/press /2018/6/5b27c2434/forced-displacement-above-68m-2017-new-global-deal-refugees-critical.html

Wallerstein I (2011) The Modern World-System I: Capitalist Agriculture and the Origins of the European World-Economy in the Sixteenth Century. Berkeley, CA: University of California Press.

Watts DJ and Dodds PS (2007) Influentials, networks, and public opinion formation. Journal of Consumer Research 34(4): 441-458.

Webster JG (2014) The Marketplace of Attention: How Audiences Take Shape in a Digital Age. Cambridge, MA: MIT Press.

Yang A and Saffer AJ (2019) Embracing a network perspective in the network society: the dawn of a new paradigm in strategic public relations. Public Relations Review 45(4): 101843.

Yang A and Taylor M (2015) Looking over, looking out, and moving forward: positioning public relations in theorizing organizational network ecologies. Communication Theory 25(1): 91-115.

Yang J, Zhang M, Shen KN, et al. (2018) Structural correlation between communities and coreperiphery structures in social networks: evidence from Twitter data. Expert Systems with Applications 111: 91-99.

\section{Author biographies}

Aimei Yang (PhD, University of Oklahoma) is an Associate Professor at the University of Southern California. Her research interests focus on civil actors' issue advocacy and issue networks. Her articles have appeared in journals such as Communication Theory, Business \& Society, Nonprofit and Voluntary Sector Quarterly, Journal of Business Ethics, and Management Communication Quarterly.

Adam J Saffer (PhD, University of Oklahoma) is an Assistant Professor at the University of Minnesota-Twin Cities. His research takes a network perspective to explore the ways individuals, groups, and organizations establish, use, and are influenced by communication networks. His research has been published in the Journal of Communication, American Behavioral Scientist, and Journal of Broadcast and Electronic Media. 